\title{
LA MENOPAUSIA EN COLOMBIA
}

\author{
Jaime Urdinola, MD.*
}

De acuerdo con las cifras proporcionadas por el DANE (1) y obtenidas del Censo sobre población de 1993, en el país existen 18 millones de mujeres, de las cuales un poco más de 4.100 .000 son mayores de 40 años en la actualidad, y cerca de 6 millones tendrán más de 50 años de edad en el año 2000. De este último número, una importante proporción estará en la menopausia al comenzar el próximo siglo sufriendo el impacto sobre su salud de los eventos asociados al climaterio. Ante esta realidad, se debe celebrar que la Revista Colombiana de Obstetricia y Ginecología reconozca la importancia del tema y publique los trabajos que aparecen en este número.

El cuidado preventivo de la mujer en esta edad incluye el ejercicio, como lo presenta la Opinión Clínica: "El Ejercicio Físico en la Postmenopausia de .....", por sus evidentes beneficios no solo sobre el hueso -en la prevención y manejo de la osteoporosis y sus secuelas-, sino también por el positivo impacto aeróbico sobre el corazón, contribuyendo a realizar la profilaxis y aliviar los problemas cardiovasculares que se presentarán con una mayor incidencia después de esta etapa de la vida.

Bernardo Moreno y Luis E. Escalante, de la Clínica Fray Bartolomé de las Casas, presentan sus resultados con el uso de un gestágeno en una investigación clínica, para detectar mujeres con patología endometrial oculta o en riesgo para desarrollarla. Contribuyen con una experiencia interesante, así su validez no haya sido aún apropiadamente establecida en estudios clínicos verdaderamente significativos (2).

La adecuada tolerancia al tratamiento hormonal, asociada al éxito terapéutico sintomático la demuestran Germán Barón, Ernesto Posada y Juan C. Ramírez en un estudio descriptivo y retrospectivo en mujeres seguidas, en el Hospital Universitario "Lorencita Villegas de Santos".

William Onatra, como coordinador de una encuesta realizada a nivel nacional, expone un panorama amplio y reciente sobre la epidemiología de la menopausia en este país.

\footnotetext{
* Secretario, Asociación Colombiana de Menopausia
}

La importancia de la comunicación de las anteriores experiencias reside en que son aportes para el cuidado de la mujer durante esta etapa, las cuales pueden servir de guía y contribuir para reducir la morbilidad potencial en años posteriores.

Queda sinembargo, un ancho y prolongado camino por recorrer, durante el cual se consulten y se de respuesta a las necesidades de las mujeres en esta etapa de la vida, no sólo de un enfoque ginecológico, sino del cuidado de todo un equipo que actúe en forma realmente interdisciplinaria. Aunque no todas las mujeres requerirán de este equipo, en nuestro país, esta tarea la realiza hasta ahora el ginecólogo, como el médico que trata, orienta y refiere las pacientes.

Dichas necesidades, y por ende futuros temas de investigación y de comunicación, se extienden desde los cambios ginecológicos que se presentan en el climaterio temprano como la hemorragia uterina disfuncional, el síndrome de tensión premestrual y la demanda por una anticoncepción efectiva y segura en este grupo de edad. Los síntomas vasomotores y otros dependientes del hipoestrogenismo se presentarán después. Todos estos cambios tendrán importante impacto en el climaterio tardío, cuando los cambios asintomáticos se manifiesten en signos preocupantes como la osteoporosis, con las secuelas que imponen fracturas y la enfermedad cardiovascular aterogénica. La asesoría y consejería oportunas sobre los cambios necesarios en el estilo de vidadieta, inactividad, cigarrillo y alcohol- contribuirán para que dichas complicaciones puedan ser evitables y reversibles en muchos casos.

En los actuales momentos, cuando la medicina en Colombia se encuentra en una profunda crisis institucional, motivada principalmente por la falta de recursos económicos, debe tomarse en cuenta que la disciplina científica en este campo no es solamente una ciencia sino la respuesta a una necesidad social. Si se consigue que esta población de mujeres adultas sean saludables y productivas, muchas de las costosas necesidades futuras de cuidado geriátrico crónico de mujeres verdaderamente enfermas pueden ser evitadas. De esta forma visionaria y previsiva, el manejo del climaterio podrá ser visto como una verdadera política nacional de salud.

\section{BIBLIOGRAFIA}

1. Censo Nacional de Población de 1993. Departamento Administrativo Nacional de Estadística (DANE).

2. Menopause and Postmenopausal Hormone Therapy. En: Speroff L, Glass RH y Kase NG, editores, Clinical Gynecologic Endocrinology and Infertility, Fifth Edition, Capitulo 18, Williams \& Wilkins, Baltimore, USA, 1994, pp 583-650. 